\title{
PLANNING OF THERMAL-HYDRAULIC TESTS OF PIPELINE SYSTEMS WITH THE METHODS OF ACTIVE IDENTIFICATION ${ }^{*}$
}

\author{
Oksana A. Grebneva ${ }^{1, \dagger}$ \\ ${ }^{1}$ Melentiev Energy Systems Institute of Siberian Branch of the Russian Academy of Sciences (ESI \\ SB RAS), Pipeline Energy Systems Department, 130, Lermontov str., Irkutsk, Russia, 664033
}

\begin{abstract}
The lack of reliable information about actual characteristics and parameters is the main deterrent to the effective application of mathematical and computer-modelling methods for solving problems of optimal reconstruction, adjustment, development of operational condition, dispatching control of pipeline systems. The paper presents the original formalization of the problems of testing pipeline systems for hydraulic and thermal losses as problems of active identification, assuming optimal planning and processing of experimental results on the basis of attracting a mathematical model of steady-state thermal-hydraulic state. The proposed technique consists in a stepwise testing strategy that ensures the extraction of maximum information with a minimum risk of excessive testing, is applicable for different types of testing of pipeline systems of arbitrary structure and configuration. Theoretically, and for an illustrative example, the potential efficiency of combining thermal and hydraulic tests of pipeline systems is shown, which is manifested in minimizing the total number of experiments to obtain a given accuracy in determining the actual characteristics of pipeline systems and the predictive properties of the model.
\end{abstract}

\section{An analysis of literature and problem statement}

The industrial methods to carry out both hydraulic and thermal tests [1-3] have some flaws: 1) by virtue of their laboriousness, the tests are normally done only for characteristic sections in terms of dates and operating conditions, which however does not guarantee a complete picture of the network state as a whole; 2) limitation on the time of the tests is related to some preparatory work to be performed before the tests (disconnection of some branches, installation of jumpers, etc.), for example, for heat network the tests are carried out during non-heating period; 3) conditions for the tests are poorly regulated; 4) the problem of placement of measuring devices, their number and mix is not formalized; 5)

\footnotetext{
${ }^{*}$ The research was carried out within the project III.17.4.3 of the Fundamental research program of SB RAS (AAAA-A17-117030310437-4) with finance support of RFBR and the Government of Irkutsk Region in the framework of research project № 17-48-380021

† Corresponding author: oksana@isem.irk.ru
} 
hydraulic and thermal characteristics are determined not for each section individually but for the groups of sections (the obtained data are extrapolated to all sections); 6)The methods do not suggest determining the resistance of the consumer that are necessary for adequate modeling of operating conditions; 7) moreover, hydraulic and thermal tests are conducted separately, which is associated with additional material, labour and time expenditure.

In order to avoid all of these problems, we propose planning of joint optimal thermalhydraulic tests that are aimed at simultaneously obtaining both hydraulic and thermophysical characteristics.

The joint thermal-hydraulic tests can potentially reduce laboriousness (decrease in the number of involved operating conditions, reduction in the number of measurements to be made) and time of the tests, as well as improve the accuracy of the parameters obtained as a result of the tests.

In many studies, such problems are solved by the methods of passive identification and mainly for the water supply systems [4-9].

The methodological framework for these studies is represented by the theory of hydraulic circuits developed at Melentiev Energy Systems Institute SB RAS [10]. The theory is considered as an intersectoral scientific and engineering discipline providing a single vector-matrix language and models to define a wide range of pipeline systems of various types and purposes. This theory was employed to formulate the task of active identification in pipeline systems and develop a new methodology [11-14]. Previously, the effectiveness of this methodology was studied when applied to the planning of hydraulic tests. The paper presents the studies on its application to plan joint thermal-hydraulic tests with an extended model of nonisothermal flow distribution.

\section{Object, goal and objectives of the research}

The primary goal of the research is to adapt the algorithms and methods for active identification to plan joint thermal-hydraulic tests of pipeline systems. The following objectives were set: 1) to modify the previously developed active identification planning method, in order to enable its application in planning of joint thermal-hydraulic tests of pipeline systems; 2) to demonstrate: a) the effectiveness of the active identification methods; b) the effectiveness of joint thermal and hydraulic tests - on a case study.

Mathematical statement of the problem. The planning of the tests is mainly aimed at influencing the major factors that affect the accuracy of the obtained parameters and characteristics of pipeline systems (hydraulic resistance coefficients and heat transfer coefficients). Moreover, for the tests to be feasible, it is necessary to take into account the constraints imposed on: 1) the state variables (including those non-fixed) that should not go beyond the technologically feasible limits after control actions; and 2) the amounts of measuring devices and sites for their possible installation. The problem is solved in the space of varied parameters.

In a general form the problem of experiment planning can be represented as follows $[11,12]$ :

$$
\min _{N, \boldsymbol{\delta}, \mathbf{X}_{\mathbf{R}}}\left\{\operatorname{det}\left[\mathbf{C}_{\boldsymbol{\alpha}}\left(\mathbf{X}_{\mathbf{R}}^{(1)}, \ldots, \mathbf{X}_{\mathbf{R}}^{(N)}, \boldsymbol{\delta}^{(1)}, \ldots, \boldsymbol{\delta}^{(N)}, \boldsymbol{\alpha}\right)\right]\right\}
$$

subject to

$$
\underline{\mathbf{R}}^{(u)} \leq \mathbf{R}^{(u)}=\left(\begin{array}{l}
\mathbf{Y}^{(u)}\left(\mathbf{X}^{(u)}\right) \\
\mathbf{X}^{(u)}
\end{array}\right) \leq \overline{\mathbf{R}}^{(u)}, q \leq l=\sum_{j=1}^{l} \delta_{j}^{(u)} \leq \bar{l}, 1<N<\bar{N}, \operatorname{det}\left(\mathbf{C}_{\boldsymbol{\alpha}}\right) \leq \bar{C}
$$


where $\operatorname{det}(\cdot)$-a determinant of the matrix; $\mathbf{C}_{\boldsymbol{a}}-$ a covariance matrix of parameters of elements; $\mathbf{R}^{(u)}$ - vector of the $u$-th state variables; $\underline{\mathbf{R}}^{(u)}$ and $\overline{\mathbf{R}}^{(u)}$ - lower and upper limits of variation in the state variables; $u$ - state index; $N$ - number of states; $\bar{N}$ - maximum admissible number of states (experiments); $\mathbf{X}_{\mathbf{R}}^{(u)}, \mathbf{Y}_{\mathbf{R}}^{(u)}-$ vectors of independent and dependent $u$-th state variables, respectively; $\boldsymbol{\delta}^{(u)}-\bar{l}$-dimensional vector of Boolean variables in the $u$-th state with components: $\delta_{j}^{(u)}=1$, if the $j$-th variable is measured in the $u$-th state, and $\delta_{j}^{(u)}=0$, otherwise , $j=1, \ldots, \bar{l} ; \boldsymbol{\alpha}$-vector of parameters of elements, $\boldsymbol{\alpha}=\{\mathbf{s}, \mathbf{k}\}$ ( $\mathbf{s}$ is vector of hydraulic resistances coefficients; $\mathbf{k}$ - vector of thermal-physical characteristics of elements - heat transfer coefficients; $\bar{l}$ - number of parameters from a set of components of vector $\mathbf{R}$, available for measurements; $q$ - minimum necessary number of measurements; $l$ - number of measurable parameters; $\bar{C}$ - a set level of accuracy.

The information criterion is represented by the determinant of the covariance matrix of parameters of elements (D-criterion) as a measure of accuracy for the estimation of vector of unknown parameters on the whole.

The overall algorithm of successive active identification of pipeline systems when planning optimal tests can be represented by the following steps: 1) planning the test conditions [15]; 2) placement of measurement devices [16]; 3) experiment; 4) processing of its results [17].

For the initial model for thermal-hydraulic tests, we propose the model of thermalhydraulic conditions [18].

In $[17,19-20]$, the authors demonstrate that despite the increased complexity of mathematical models of thermal-hydraulic conditions, they manage to much faster redefine (compared to the models of isothermal flow distribution), owing to the involvement of temperature measurements.

\section{Case study}

The case study aims to demonstrate the advantages of the joint thermal-hydraulic tests over separate ones. To this end, we set the following objectives: 1) to perform a cycle of calculations on a concrete example in order to conduct different types of tests with the use of various mathematical models to check the effect of the active identification algorithm; 2) to study the influence of the criterion choice on the planning results; 3) to investigate the impact of conditions on the optimal choice of measuring devices

The studies were conducted for a conventional pipeline system depicted in Figure 1.

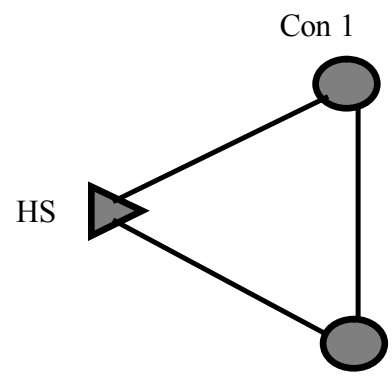

Con 2

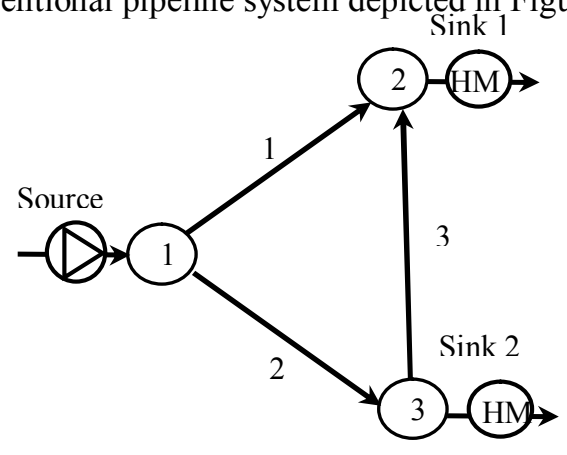

HS - heat source; Con 1, Con 2 - consumers 1 and 2, respectively; HM - heat meter.

Fig. 1. A block diagram and a calculation scheme of the conventional pipeline system. 
Calculation conditions: Pressure at the first node is considered to be fixed. . For the variables that could be measured we assumed the following values of standard deviations. To plan the first experiment, it is necessary to specify the initial approximation of the resistance and heat transfer coefficients values and their standard deviation. The rule of their setting by expert is presented in [21].

To analyze the results of the numerical experiment we specified the limiting values according to the used criterion: for hydraulic parameters $-\bar{C}_{C_{s}}=8.0 \cdot 10^{-27}$; and for thermophysical parameters $-\bar{C}_{C_{k}}=1.0 \cdot 10^{-6}$.

The problem-solving process can be stopped only by the estimation results, therefore with the constraint imposed on the determinant of the covariance matrix of parameters of elements the question arises about how to impose this constraint and how to assess the quality of the data obtained from the tests based on the constraint. Consequently, in the process of the numerical studies, it was decided to introduce an additional criterion determined as $\gamma=\max _{j} \frac{\hat{\sigma}_{j}^{2}}{\tilde{\sigma}_{j}^{2}}$ (where $\hat{\sigma}_{j}^{2}$ - variance of unmeasured state variables that was obtained from the estimation; $\tilde{\sigma}_{j}^{2}$ - variance of direct measurement of the state variables).

Criterion $\gamma$ demonstrates the relation between the projected variance and the variance of direct measurement. Thus, the criterion makes it possible to characterize the adequacy of the applied pipeline system models.

Planning of the state for tests. The problem is large dimensional, nonlinear and multiextremal. The authors of [15] propose a solution to this problem by the widely accepted genetic algorithms. The solution is reduced to the guided search with elements of "stochastic walk" with the help of selection operators. As the final result, some subset of points with the minimum deviation of the objective function is formed.

Planning of the state for tests makes it possible to determine an optimal composition of the vector of varied parameters, which leads to extraction of maximum information on the whole system.

Dependent variables of model [18] included vectors of: nodal flow rates, flow rates in the branches, temperatures at the beginning and end of the branches and temperatures of mixed flows at nodes and temperature of sinks $\mathbf{Y}=\left(\mathbf{Q}^{\mathrm{T}}, \mathbf{x}^{\mathrm{T}}, \mathbf{t}_{b}^{\mathrm{T}}, \mathbf{t}_{\text {end }}^{\mathrm{T}}, \mathbf{T}^{\mathrm{T}}\right)^{\mathrm{T}}$. The independent variables of model [18] were represented by vectors of nodal pressures, element variables and temperature of source $\mathbf{X}=\left(\mathbf{P}^{\mathrm{T}}, \boldsymbol{a}^{\mathrm{T}}, \mathbf{T}_{\mathbf{s}}^{\mathrm{T}}\right)^{\mathrm{T}}$. Vector of element variables was $\boldsymbol{\alpha}=\{\mathbf{s}, \mathbf{k}\}$. Full vector of measured variables consisted of vectors of nodal pressures, nodal flow rates, flow rates in the branches and vectors of source temperatures, temperatures at the beginning and end of the branches $\mathbf{Z}_{1}=\left(\mathbf{P}^{\mathrm{T}}, \mathbf{Q}^{\mathrm{T}}, \mathbf{x}^{\mathrm{T}}, \mathbf{t}_{b}^{\mathrm{T}}, \mathbf{t}_{\text {end }}^{\mathrm{T}}, \mathbf{T}_{s}^{\mathrm{T}}\right)^{\mathrm{T}}$.

After the a priori information had been specified the state was designed. It was supposed that the temperature of source and sinks at consumers 1 and 2 that played a role of varying state variables could be controlled. The ranges of variation in the variables were: $290 \leq Q_{2} \leq 490,10 \leq Q_{3} \leq 210,70 \leq T_{s} \leq 95$. For example, some arbitrary permissible state satisfying these constraints with the parameters: $T_{s}=70, Q_{2}=290, Q_{3}=10$ has $\gamma_{\max }=1.28861$. Using the methodology of active identification proposed above, we obtained a state $\left(T_{s}=95, Q_{2}=340, Q_{3}=60\right)$ with $\gamma_{\max }=0.99999675 \approx 1$. It can be seen that the criterion $\gamma_{\max }$ in obtained optimal state is $30 \%$ better than in the permissible state. 
In this case, the accuracy of estimating the immeasurable parameters is commensurable with their direct measurement.

Planning of optimal placement of meters for pipeline system tests. Formation of an optimal set of meters is based on the algorithm described in [13]. The algorithm is intended to sequentially reduce a maximum admissible set of meters by removal of the measurement that would degrade the information criterion to the minimum extent. This algorithm allows the global solution to be obtained in the finite limited number of steps.

The calculation results for the planned thermal-hydraulic condition are presented in Table 1 .

Table 1. Optimal placement of meters in planned condition.

\begin{tabular}{|c|c|c|c|c|c|c|c|}
\hline $\mathrm{RM}$ & $\operatorname{det}\left(\mathbf{C}_{\alpha}\right)$ & $\mathrm{RM}$ & $\operatorname{det}\left(\mathbf{C}_{\alpha}\right)$ & $\mathrm{RM}$ & $\operatorname{det}\left(\mathbf{C}_{\alpha}\right)$ & $\mathrm{RM}$ & $\operatorname{det}\left(\mathbf{C}_{\alpha}\right)$ \\
\hline \multicolumn{8}{|c|}{ Initial composition of measurement vector } \\
\hline$Q_{1}$ & $0.8165 .10^{-41}$ & $t_{b 2}$ & $0.0825 .10^{-40}$ & $t_{\text {end } 1}$ & $0.0921 .10^{-40}$ & $t_{\text {end } 2}$ & $0.1324 .10^{-34}$ \\
\hline$P_{2}$ & $0.9314 .10^{-41}$ & $t_{b 1}$ & $0.1795 .10^{-39}$ & $x_{3}$ & $0.1692 .10^{-37}$ & $t_{\text {end } 3}$ & $0.6844 .10^{-31}$ \\
\hline$P_{3}$ & $0.1031 .10^{-40}$ & $t_{b 3}$ & $0.1516 .10^{-39}$ & & & & \\
\hline \multicolumn{8}{|c|}{ Optimal composition of measurement: $P_{1}, Q_{2}, Q_{3}, T_{s}, T_{\text {sink } 2}, T_{\text {sink } 3}, x_{1}$} \\
\hline
\end{tabular}

*RM - removed measurement.

As is seen from the Table, the conditions have a different optimal set of meters. However, the algorithm enables us to terminate the problem solution at any step and to select the variant of a set of meters with their required number and minimum loss in accuracy.

Effective of use of a thermal-hydraulic model for planning the tests of heating networks. The solution results of the problem of conditions planning for different types of tests show that in the planning of hydraulic tests the condition with the optimal criterion value does not coincide with the condition in the planning of thermal tests.

The solution results also reveal that the target accuracy level in running hydraulic tests on hydraulic condition model can be achieved using 4 conditions. The use of additional information on temperatures in the hydraulic tests on model of thermal-hydraulic conditions [18] enables a decrease in the number of planned conditions to 1 and improvement in an evaluation accuracy of hydraulic parameters.

In the thermal tests on thermal condition model, the required level of evaluation accuracy of thermophysical parameters can be achieved on the basis of 2 planned conditions.

In the joint thermal-hydraulic tests only one condition is planned. The results show that the use of $\operatorname{det} C_{s k}$ as an information criterion provides for a high accuracy of both hydraulic and thermophysical parameters of pipeline systems. Hence, from the viewpoint of reduction in the number of planned conditions, and improvement of evaluation accuracy of both hydraulic and thermophysical parameters of pipeline systems it is preferable to carry out joint thermal-hydraulic tests.

\section{Conclusions}

The research is focused on the specific features of planning of joint thermal-hydraulic conditions. 
The algorithm of planning of the state for tests and optimal placement of meters is tried out using the nonisothermal flow distribution model.

Possible reduction of the experiments is exemplified numerically, which potentially leads to: 1) reduction in labor intensity; 2) improvement in accuracy against the traditional approaches.

The study demonstrates that the proposed algorithm allows the planning of tests using different flow distribution models.

\section{References}

1. RD34.20.519-97 (Service of Excellence, Moscow, 1997) [in Russian]

2. RD34.09.255-97 (Service of Excellence, Moscow, 1998) [in Russian]

3. RD 153.34.1-20.526-00 (Open Joint-Stock Company "VTI", 2001) [in Russian]

4. Srinivasa Lingireddy, Lindell E. Ormsbee, Civil Engineering and Environmental Systems, 19(1), 13 (2012)

5. S. Alvisi, E. Creaco, M. Franchini, Urban Water Journal, 8 (4), 203 (2011)

6. Guoping Yu., Roger S. Powell, Intern. Journal of Systems Science, 25 (12), 2155 (1994)

7. Ilyas Eker, Tolgay Kara, ISA Transactions, 42 (3), 461 (2003)

8. B. Coulbeck and C.H. Orr, Civil Engineering Systems, 1, 139 (1984)

9. Alireza Yazdani and Paul Jeffrey, Interdisciplinary Journal of Nonlinear Science, 21 (1), $11(2011)$

10. A. P. Merenkov and V. Ya. Khasilev, The Theory of Hydraulic Circuits (Science, Moscow, 1985) [in Russian]

11. O. A. Grebneva, Candidate's Dissertation in Engineering (ESI SB RAS, Irkutsk, 2005)

12. O.A. Grebneva, N.N. Novitsky, Pipeline systems of power engineering, 177 (Science, Novosibirsk, 2007) [in Russian]

13. O.A. Grebneva, N.N. Novitsky, Thermal Engineering, 61 (10), 754 (2014)

14. O.A. Grebneva, N.N. Novitsky, Pipeline Energy Systems: Methodological and Applied Problems of Mathematical Modelling, 220 (Science, Novosibirsk, 2015) [in Russian]

15. O.A. Grebneva, System research in power engineering, 48 (ESI SB RAS Irkutsk, 2001) [in Russian]

16. O.A. Grebneva, N.N. Novitsky, Thermal Engineering, 61 (9), 685 (2014)

17. N.N. Novitsky, Estimation of the parameters of hydraulic circuits (Science, Novosibirsk, 1998) [in Russian]

18. N.N. Novitsky, V.V. Tokarev, Thermophysics and Aeromechanics, 14 (2), 289 (2007) [in Russian]

19. N.N. Novitsky, Candidate's Dissertation in Engineering (ESI SB RAS, Irkutsk, 1986). [in Russian]

20. N.N. Novitsky, V.G. Sidler, Izv. of AS of USSR. Energy and transport, 4, 155 (1984) [in Russian]

21. O.A. Grebneva, N.N. Novitsky, Pipeline systems of power engineering, 245 (2008) [in Russian] 